\title{
Anomalous diffusion of discrete solitons driven by evolving disorder
}

\author{
Zhi-Yuan Sun ${ }^{1,2, *}$ and $\mathrm{Xin} \mathrm{Yu}^{1, \dagger}$ \\ ${ }^{1}$ Institute of Fluid Mechanics, Beihang University, Beijing 100191, China \\ ${ }^{2}$ International Research Institute for Multidisciplinary Science, Beihang University, Beijing 100191, China
}

(Received 11 February 2020; revised manuscript received 6 May 2020; accepted 2 June 2020; published 23 June 2020)

\begin{abstract}
Anomalous diffusion is simulated in this paper by studying the transport of discrete solitons in a lattice with evolving disorder. We find a Richardson-type diffusion for the small solitons and a regime of transient diffusion for larger solitons within the ensemble-averaged description. As a comparison, the time-averaged observables present a ballistic scaling for both cases. However, distribution of these observables changes remarkably with the soliton size. Our results suggest violation of ergodicity for the solitons' diffusive processes, which are expected to shed light on further understanding of the discreteness-disorder-nonlinearity interaction.
\end{abstract}

DOI: 10.1103/PhysRevE.101.062211

\section{INTRODUCTION}

Discrete solitons (DSs), the self-trapped wave packets balancing lattice dispersion and nonlinearity, have been observed in a variety of physical systems such as optics, Bose-Einstein condensates, and crystals [1-5]. One of the theoretical efforts is dedicated to the understanding of DS mobility in ordered lattices [6-9], where some prototype forms of discrete nonlinear Schrödinger (DNLS) models have been considered by using the concept of the Peierls-Nabarro (PN) potentials [10,11]. However, study on the motion of DSs for disordered lattices appears to be limited [12]. This should be an interesting topic concerning the interplay among discreteness, nonlinearity, and randomness that is on the other hand different from the wave-packet spreading induced by weak nonlinearity [13-15]. Recently, it was reported that the PN landscape could be effective in analyzing the DS mobility in statically (only spacedependent) disordered potential [16], and the breathing DSs experience a short-time transient diffusion and consequently, a longer-time localization [17]. A further question then arises: How is the transport of DSs in a lattice with evolving (both space- and time-dependent) disorder? Our investigation in this work is expected to provide some insights into such a problem.

For the wave-packet spreading (width expansion) described by a linear Schrödinger equation with evolving disorder, the optical experiment [18] as well as theoretical study [19] suggest a transport faster than ballistic (we call it hyperdiffusion), while the diffusion rate may depend on the correlation properties of the relevant random potential $[20,21]$. The

\footnotetext{
*sunzhiyuan137@aliyun.com

†yuxin@buaa.edu.cn
}

Published by the American Physical Society under the terms of the Creative Commons Attribution 4.0 International license. Further distribution of this work must maintain attribution to the author(s) and the published article's title, journal citation, and DOI. diffusive expansion of wave packets with evolving disorder also can be found in binary kagome ribbons [22] and ultracold atomic systems with noise and interaction [23]. Nevertheless, for the highly localized DSs, we might be interested in their particlelike nature, considering random walks of the DS's center-of-mass accordingly. In such a vein, former works have studied for continuous systems the Brownian motion of solitons [24,25], transport of nonlocal solitons [26-28], as well as the Anderson localization of solitons [29,30]. Due to addition of the discreteness, the transport of DSs would present more sophisticated and interesting visions as revealed hereafter.

\section{DISCRETE NONLINEAR SCHRÖDINGER MODEL AND ADIABATIC APPROXIMATION}

In this paper we consider the one-dimensional DNLS model, written as

$$
i \frac{\partial \psi_{n}}{\partial t}=-\left(\psi_{n-1}+\psi_{n+1}\right)-v\left|\psi_{n}\right|^{2} \psi_{n}+\epsilon_{n}(t) \psi_{n},
$$

where $\psi_{n}$ is the amplitude at the site $n$ and time $t$, and $v$ measures the strength of the cubic nonlinearity. The random potential $\epsilon_{n}(t)$ is assumed to be Gaussian with zero mean, and $\delta$ correlated both in space and in time, of the form

$$
\begin{aligned}
\left\langle\epsilon_{n}(t)\right\rangle & =0, \\
\left\langle\epsilon_{n}(t) \epsilon_{n^{\prime}}\left(t^{\prime}\right)\right\rangle & =\sigma^{2} \delta_{n n^{\prime}} \delta\left(t-t^{\prime}\right),
\end{aligned}
$$

where the angular brackets stand for statistical averaging. Equation (1) may be viewed as a model describing the light propagation in nonlinear waveguides with evolving disorder or the evolution of cold atomic wave packets in dynamically random potential [31,32].

When the random potential is weak and the DS velocity is slow, an adiabatic approximation assumes the form of the DS moving in the lattice governed by Eq. (1) as

$$
\psi_{n}(t)=\sqrt{\frac{2}{v}} \frac{\sinh (\mu)}{\cosh [\mu(n-x)]} \exp [i k(n-x)+i \alpha],
$$


where the parameter $\mu$ relates to the inverse of the DS width, and $x$ is the DS's center of mass. Note that although Eq. (3) is not an exact solution of Eq. (1) when $\epsilon_{n}(t)$ is vanished, it can be viewed as a good approximation especially for $\mu<1$ (a detailed justification is presented in Appendix A).

Following the similar perturbation procedure as in [16] (some detailed interpretations are presented in Appendix B), we have $\mu$ being constant and two evolution equations for the DS's movement:

$$
\begin{aligned}
& \frac{d x}{d t}=\frac{2 \sinh (\mu)}{\mu} \sin (k), \\
& \frac{d k}{d t}=-\frac{8 \pi^{3} \sinh ^{2}(\mu)}{\mu^{3} \sinh \left(\pi^{2} / \mu\right)} \sin (2 \pi x)+f(x, t),
\end{aligned}
$$

where the equivalent randomness-generated force $f(x, t)$ reads

$$
f(x, t)=-\sum_{n=-\infty}^{+\infty} \frac{\epsilon_{n}(t) \sinh ^{2}(\mu) \tanh [\mu(n-x)]}{\cosh [\mu(n-x-1)] \cosh [\mu(n-x+1)]} .
$$

Generally speaking, this force $f(x, t)$ is a nonstationary random process in space with its correlation function depending on both $x$ and $\Delta x=x-x^{\prime}$ [16]. Besides, the first nonlinear term in Eq. (4b), the discreteness-generated force, may also bring considerable complexity to these equations.

\section{SMALL DISCRETE SOLITONS AND RICHARDSON-TYPE DIFFUSION}

When $\mu \ll 1$, we may have two approximations for small DSs: (i) The random process $f(x, t)$ can be viewed to be stationary in both space and time [16], and its correlation function reads

$$
\left\langle f(x, t) f\left(x^{\prime}, t^{\prime}\right)\right\rangle=\sigma^{2} F(\mu) R\left(x-x^{\prime}\right) \delta\left(t-t^{\prime}\right),
$$

where $F(\mu)=3 \operatorname{coth}(\mu)-\tanh (\mu)-3 / \mu$, and $R(|x|)$ falls off to zero as $|x| \rightarrow \infty$ [we have the value $R(0)=1$ ]. (ii) The discreteness-generated force is several orders of magnitude smaller than the randomness-generated force, such that it can be reasonably neglected from Eq. (4b). Hence, considering the slow motion of the DSs (the parameter $k \ll 1$ ), we derive the following simplified equation for the center of mass:

$$
\frac{d^{2} x}{d t^{2}}+\eta(x, t)=0
$$

where $\langle\eta(x, t)\rangle=0$ and $\left\langle\eta(x, t) \eta\left(x^{\prime}, t^{\prime}\right)\right\rangle=\sigma^{2} G(\mu) R(x-$ $\left.x^{\prime}\right) \delta\left(t-t^{\prime}\right)$, with $G(\mu)=4 \sinh ^{2}(\mu) F(\mu) / \mu^{2}$. Note that Eq. (7) is generally valid in describing the DS motion for considerably long time if the random potential is weak enough, and the radiation from DS to the surrounding environment can be negligible (a brief comment on the radiation and nonlinearity is presented in Appendix C).

\section{A. Ensemble-averaged description}

Equation (7) is nontrivial to integrate since $\eta(x, t)$ is also a function of the trajectory $x$. However, by assuming that $x(t)$ and $\eta(x, t)$ are independent random quantities (this assumption holds hereafter when the relevant formulas are analytically calculated), the following ensemble-averaged mean squared displacement (eMSD) can be calculated exactly [33]:

$$
\left\langle x^{2}(t)\right\rangle=\frac{1}{3} G(\mu) \sigma^{2} t^{3} .
$$

The validation of Eq. (8) has been checked by numerically integrating Eq. (7) for typical parameters (not shown here). Generally speaking, the eMSD $\left\langle x^{2}(t)\right\rangle \sim t^{\gamma}$ characterizes the feature of either a Brownian motion with $\gamma=1$ or an anomalous diffusion with $\gamma \neq 1$ for a certain timescale. Different diffusive regimes usually include subdiffusion with $0<\gamma<$ 1 , superdiffusion with $1<\gamma<2$, ballistic diffusion with $\gamma=$ 2 , and a faster hyperdiffusion with $\gamma>2$.

This hyperdiffusive scaling (8) of the order $\sim t^{3}$ corresponds to the Richardson diffusion [36], which also describes the celebrated Gordon-Haus effect of continuous solitons in optical fiber [37]. A statistical mechanism suggested by Kolmogorov and Obukhov explained Richardson scaling by the Newtonian dynamical system driven by a Gaussian $\delta$ correlated noise [38,39], which was similar as in Eq. (7). On the other hand, Richardson diffusion of quantum wave packets was reported in random potentials [21,40], and this scaling was also found for turbulent diffusion of particles [41], for a phase of the cold-atom diffusion in optical lattices [42] and for a transport inside neurons as well [43].

To verify the result (8), we directly performed numerical simulation of Eq. (1). The time domain was uniformly discretized with a step size $\Delta t=0.01$, and the random potential $\epsilon_{n}(t=j \Delta t)$ was assigned Gaussian-distributed random numbers with zero mean and standard deviation $\sigma / \sqrt{\Delta t}$. We chose a coordinate moving with the center of mass of the DS and used periodic boundary conditions for more than $N=600$ sites. The three-part split symplectic scheme of order 2 (type $\mathrm{ABC}^{2}$ ) [44-46] was employed to integrate Eq. (1) for each step size, while a fourth-order Runge-Kutta method was also utilized for double checking our numerical results. The simulation was implemented up to the time $T=10^{6}$, and the statistical quantities hereafter were all derived by averaging over $M=200$ realizations of the random potentials. For the initial conditions, we considered the stable on-site DSs constructed by a Newton-Raphson iteration method [2,3], with their amplitudes denoted as $A=\max \left(\left|\psi_{n}\right|\right)$. The simulated center of mass $x(t)$ were obtained as $x(t)=\sum_{n} n\left|\psi_{n}\right|^{2} / P$, where $P=\sum_{n}\left|\psi_{n}\right|^{2}$ is the conserved mass of the DSs.

In Fig. 1 we present the simulation results for $A=0.5$. Panel (a) shows a comparison of the initial DS profile with the averaged profile at $t=10^{6}$, where we see that the DSs are well preserved for long-time propagation, with the radiation much smaller than the core of the DSs (at least three orders of magnitude smaller in amplitudes). Panel (b) displays the trajectories for different samples, and the averaged speed of DSs is not beyond the level $\sim 0.1$. These features further support our assumptions of weak radiation and small velocity. A very good agreement between Eq. (8) and the numerical results can be seen in panel (c).

\section{B. Time-averaged observable}

We now look at the Richardson-type diffusion of DSs in the sense of single particle tracking, for which a typical time-averaged mean squared displacement (tMSD) over an 

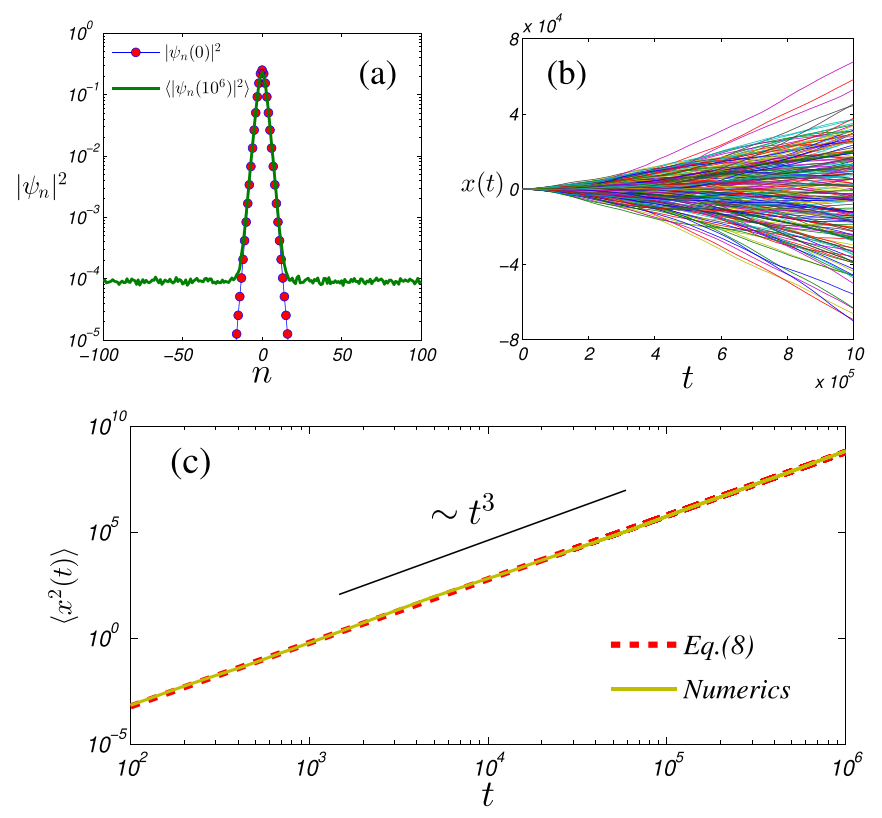

FIG. 1. (a) Comparison of the DS profiles $\left|\psi_{n}\right|^{2}$ at $t=0$ (red circles) and $t=10^{6}$ (green solid line), with the latter averaged out from 200 independent realizations. (b) Motion trajectories $x(t)$ as functions of $t$ for 200 realizations of the random potentials. (c) Comparison of the scaling law $\left\langle x^{2}\right\rangle \sim t^{3}$ between Eq. (8) (red dashed line) and the numerical results (yellow solid line). The parameters are $\mu=0.35, \sigma=0.0002$, and $v=1$.

individual trajectory is given by

$$
\overline{\delta^{2}(\Delta)}=\frac{1}{T-\Delta} \int_{0}^{T-\Delta}[x(t+\Delta)-x(t)]^{2} d t
$$

where $\Delta$ is the lag time defining a time window slid along the trajectory $x(t)$, and $T$ is the overall measurement time [47-49]. A stochastic process is called ergodic when the tMSD is observed converging to the eMSD in the limit of long measurement time, i.e., $\lim _{\Delta / T \rightarrow 0} \overline{\delta^{2}(\Delta)}=\left\langle x^{2}(\Delta)\right\rangle$, with the identification $t \leftrightarrow \Delta$. The Brownian motion (normal diffusion) is a typical example for ergodic processes where both the eMSD and tMSD display a linear growth with $t$ or $\Delta$ [47-49]. Oppositely, this equality is violated for a nonstationary process and we find the phenomenon of so-called weak ergodicity breaking: $\lim _{\Delta / T \rightarrow 0} \overline{\delta^{2}(\Delta)} \neq\left\langle x^{2}(\Delta)\right\rangle$ [47-54]. Different examples of anomalous diffusion exhibiting their nonergodic behaviors have been listed in a review work by Metzler $e t$ al. [47].

For nonergodic processes, the tMSDs vary from trajectory to trajectory and show an obvious amplitude scatter (such a scatter may be observed even for ergodic processes with finite measurement times). We can therefore consider the averaged tMSD over the ensemble of $M$ different trajectories [47-50],

$$
\left.\overline{\left\langle\delta^{2}(\Delta)\right.}\right\rangle=\frac{1}{M} \sum_{j=1}^{M} \overline{\delta_{j}^{2}(\Delta)},
$$

which by using Eqs. (7) and (9) then goes as

$$
\left\langle\overline{\delta^{2}(\Delta)}\right\rangle=\frac{1}{2} G(\mu) \sigma^{2} T \Delta^{2}
$$

for $\Delta / T \rightarrow 0$. The disparity between the eMSD (8) and the tMSD (11) may indicate the occurrence of weak ergodicity breaking for the Richardson-type diffusion of DSs.

The scatter of the tMSDs $\overline{\delta^{2}(\Delta)}$ around their mean $\left\langle\overline{\delta^{2}(\Delta)}\right\rangle$ can be quantified in terms of the probability density function (PDF) $\phi(\xi)$ of the dimensionless variable $\xi=$ $\overline{\delta^{2}(\Delta)} /\left\langle\overline{\delta^{2}(\Delta)}\right\rangle$. The variance of $\xi$ is further defined as the ergodicity breaking (EB) parameter $[47,48,50]$

$$
\mathcal{E}(\Delta)=\left\langle\xi^{2}\right\rangle-1
$$

which is widely used to measure the trajectory-to-trajectory fluctuations in single particle tracking. This parameter can be studied in the limit $\Delta / T \rightarrow 0$, and it has been also used to qualify the spread of $\xi$ for finite ratio $\Delta / T$ [55,56]. For Brownian motion, the ergodicity breaking parameter has the scaling $\mathcal{E}(\Delta)=4 \Delta /(3 T)$ at $\Delta / T \rightarrow 0$, while for certain anomalous diffusion the EB parameters have finite values even in the limit $\Delta / T=0[47,50,55,56]$. In our work, after some calculation by using Eqs. (7), (9), and (12), we derive the following EB parameter for $\Delta / T \rightarrow 0$ :

$$
\mathcal{E}(\Delta) \simeq \frac{4}{3}
$$

This result means that for long measurement times the tMSDs remain random variables with almost a constant scatter, and the individual time-averaged trajectory cannot be reproduced from another one, which characterizes the nonergodic feature of the DS spreading.

Figure 2 compares the data from directly simulating Eq. (1) of the DS motions with the theoretical results Eqs. (11) and
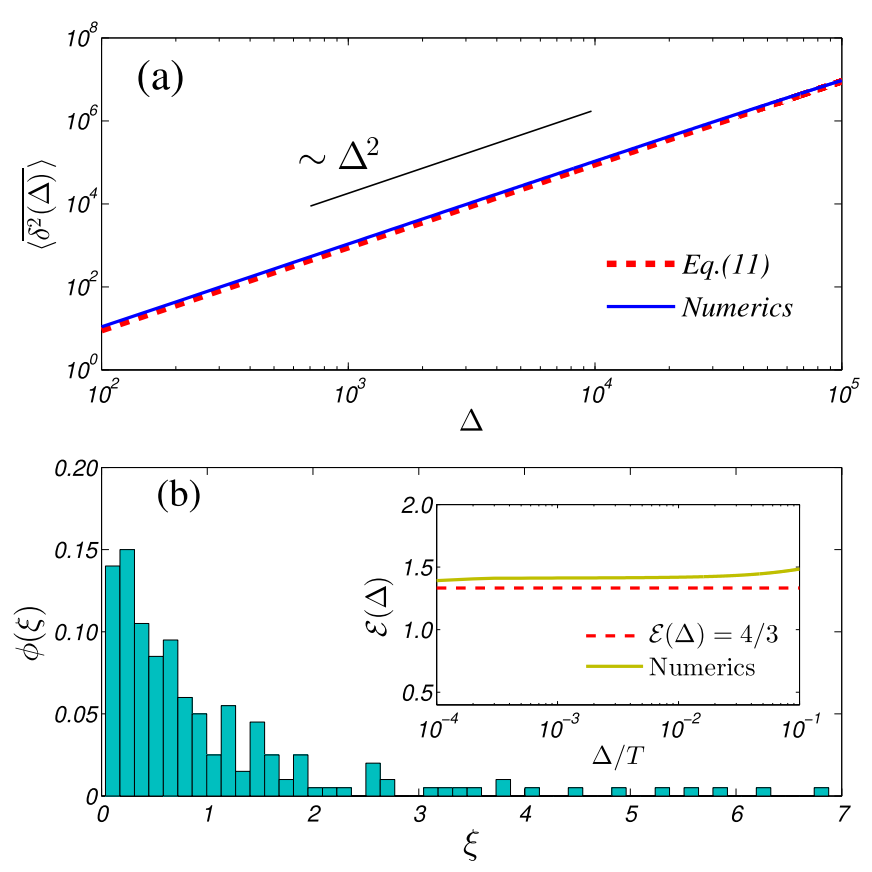

FIG. 2. (a) Comparison of the scaling law $\left\langle\overline{\delta^{2}(\Delta)}\right\rangle \sim \Delta^{2}$ between Eq. (11) (red dashed line) and the numerical results (blue solid line). (b) Distribution of the normalized tMSDs in terms of the $\operatorname{PDF} \phi(\xi)$ at $\Delta=10^{4}$. The embedded panel shows the ergodicity breaking parameters $\mathcal{E}(\Delta)$ as functions of $\Delta / T\left(T=10^{6}\right)$, where the red dashed line denotes Eq. (13) and the yellow solid line displays the simulation results. The parameters are the same as those in Fig. 1. 

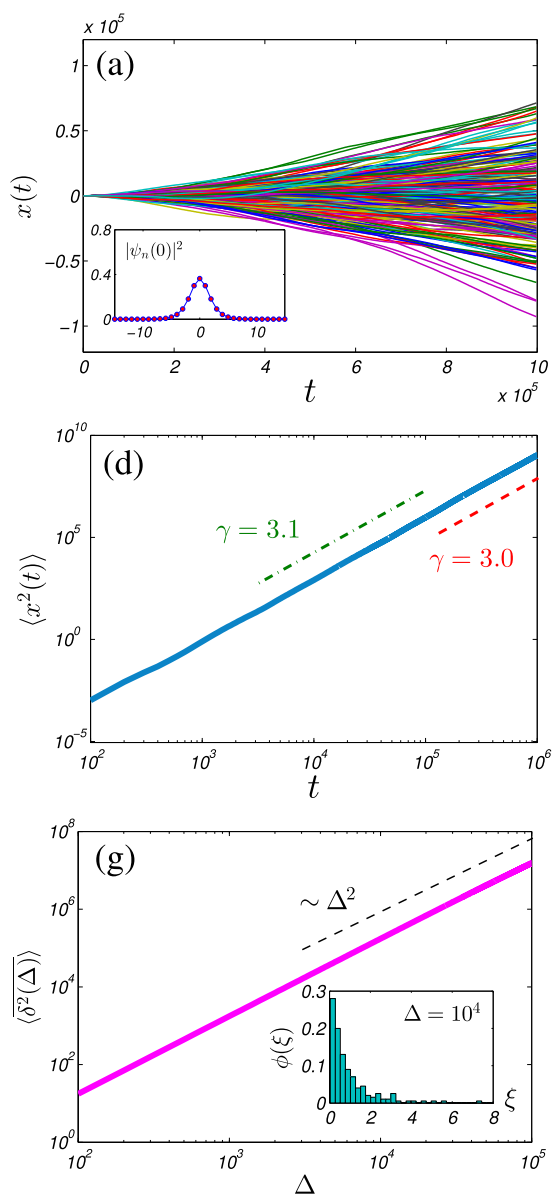
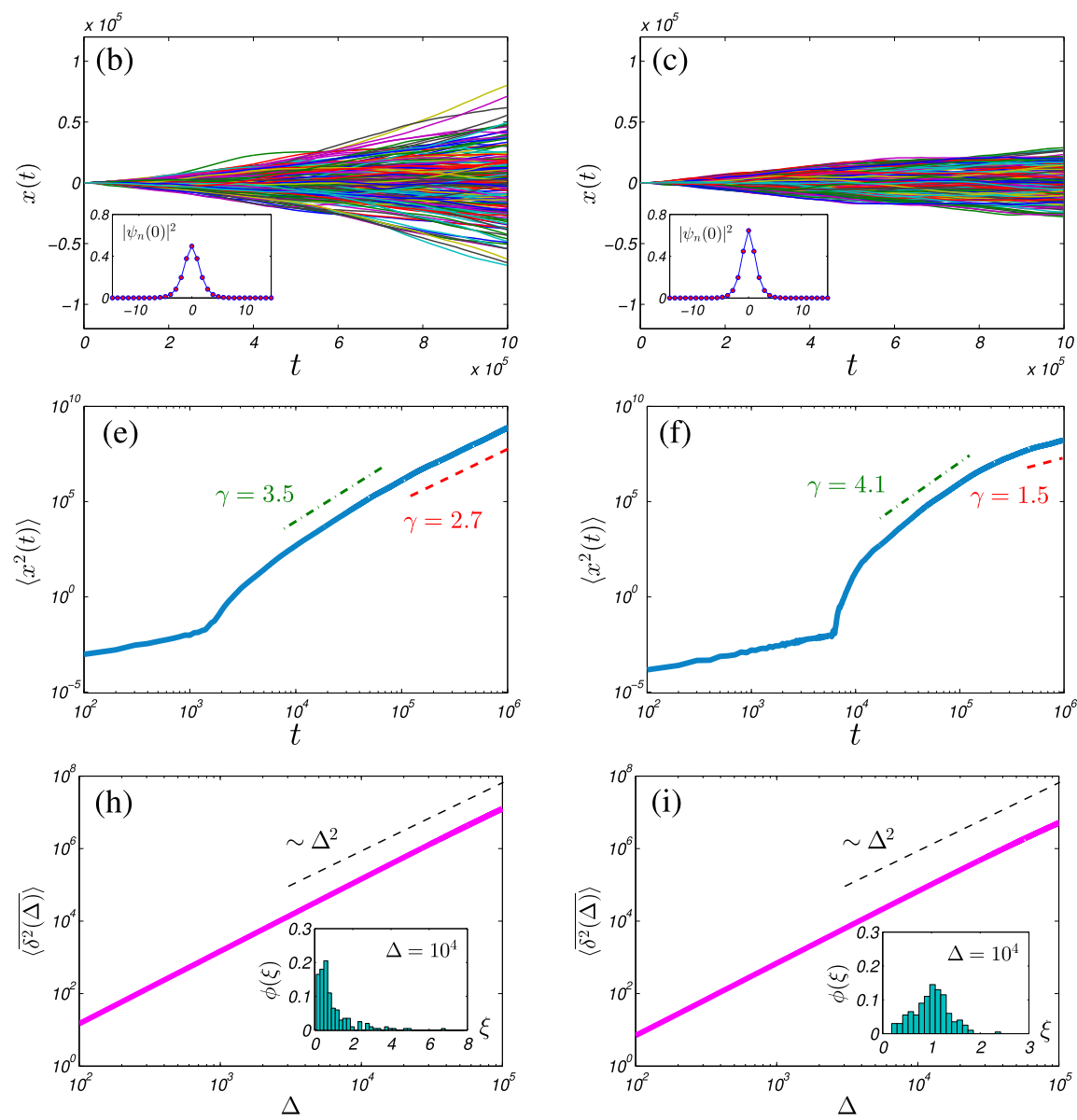

FIG. 3. (a)-(c) The DS trajectories $x(t)$ as functions of $t$ for 200 realizations of the random potentials for $A=0.60,0.70$, and 0.80 . The embedded panels show the initial DS profiles $\left|\psi_{n}(0)\right|^{2}$. (d)-(f) The eMSD $\left\langle x^{2}(t)\right\rangle$ as functions of $t$ for cases (a)-(c). The scaling exponent $\gamma$ is obtained by fitting the data in each time interval with $R^{2}>0.996$. (g)-(i) The trajectory-averaged tMSD $\left\langle\overline{\delta^{2}(\Delta)}\right\rangle$ as functions of $\Delta$ for cases (a)-(c). The embedded panels display the PDF $\phi(\xi)$ at $\Delta=10^{4}$ (the distribution $\phi(\xi)$ at different $\Delta$ keeps a similar profile for each case). Other parameters include $v=1$ and $\sigma=0.0002$.

(13), which shows reasonably good agreements. Additionally, we also plot the PDF $\phi(\xi)$ for $\Delta=10^{4}$ in Fig. 2(b) [the distribution $\phi(\xi)$ for smaller $\Delta$ keeps almost the same profile]. It is observed that the maximum of $\phi(\xi)$ is not centered at the value $\xi=1$, and $\phi(\xi)$ becomes highly asymmetric even for small $\Delta / T$, which is far different from the Gaussian distribution of ergodic Brownian motion [56]. On the other hand, the long tail of $\phi(\xi)$ with $\xi$ deviating from the unity indicates some extreme events apparently contributing to the trajectory-averaged tMSD.

\section{LARGER DISCRETE SOLITONS AND TRANSIENT DIFFUSION}

In this section, we shall investigate the transport for larger DSs (corresponding to larger $\mu$ ), where the approximation of Eq. (7) is invalid mainly due to the interplay between the discreteness and randomness. Our discussions are based on the numerical simulations of Eq. (1) for $A \approx 0.6-0.8$. The results are provided in Fig. 3.

We see in Figs. 3(a)-3(c) that the transverse diffusion seems to be progressively suppressed for larger DS up to the time $t=10^{6}$. However, the details are more complicated.
Figures 3(d)-3(f) show that after the DS has a substantial displacement (usually with $\left\langle x^{2}(t)\right\rangle \gtrsim 10^{0}$ such that the soliton center moves at least one site long statistically), the time evolution of the eMSD on longer accords with the Richardsontype diffusion but presents a transient diffusion which may be divided into different time intervals: an early period of hyperdiffusive regime with $\gamma>3.0$, and a longer-time regime with $\gamma<3.0$ where slower diffusion may occur for the larger DS. We stress that the regime of transient behavior was discussed within our finite computational limit up to $T=10^{6}$. The transition between different spreading states can also be found in other systems, for instance, see the diffusion of telomeres in mammalian cells (from subdiffusion to normal diffusion) [57], and the transport in driven Brownian ratchets (from superdiffusion to subdiffusion) [58,59]. However, the spreading speed of the DSs reported in this work appears to be much faster for the above transient time regimes.

In Figs. 3(g)-3(i) we plot the time-averaged description for this transient diffusion. We are interested in the trajectoryaveraged tMSDs that are not deviating from the scaling of $\sim \Delta^{2}$ except for the large lag times $\Delta$ approaching $T / 10$. Nevertheless, the distribution $\phi(\xi)$ changes remarkably with the DS amplitude. The scatter of the individual tMSDs $\overline{\delta^{2}(\Delta)}$ 

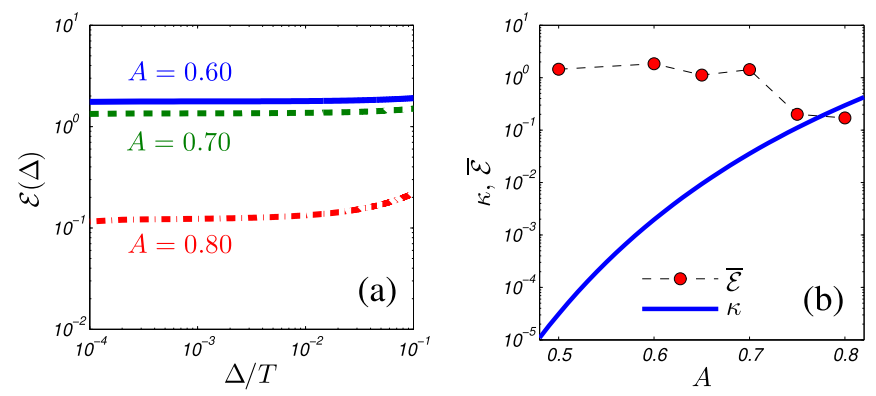

FIG. 4. (a) The ergodicity breaking parameters $\mathcal{E}(\Delta)$ as functions of $\Delta / T$ for $A=0.60,0.70$, and $0.80\left(T=10^{6}\right)$. (b) The parameter $\kappa$ as a function of $A$ ( $\mu$ is derived by fitting the DS similarly as in Appendix A), and typical plots of $\overline{\mathcal{E}}$ for six different amplitudes $A$. Other parameters are the same as in Fig. 3.

becomes progressively smaller and symmetric as the DS amplitude increases [see the embedded panels in Figs. 3(g)-3(i)]. Consequently, for larger DSs the tMSDs and the ergodic properties appear be less dominated by few extreme trajectories in the data ensembles, which is different from the case of Richardson diffusion.

To further understand the dependence of the scatter on DS size, we show the evolution of the parameters $\mathcal{E}(\Delta)$ with $\Delta$ for the above cases, as seen in Fig. 4(a). This figure suggests that $\mathcal{E}(\Delta)$ can also be approximated as constants for relatively larger DSs (especially for $\Delta / T \ll 1$ ), and their values apparently drop down for about one order of magnitude when the DS amplitude increases to $A \approx 0.80$. We define the following averaged EB parameter to characterize the overall level of the scatters within the interval $[0, \tau]$ :

$$
\overline{\mathcal{E}}=\frac{1}{\tau} \int_{0}^{\tau} \mathcal{E}(\Delta) d \Delta,
$$

where $\tau=T / 10$ hereby. Figure 4(b) gives several typical plots of $\overline{\mathcal{E}}$ for increasing amplitudes of the DSs, where we see that a crossover actually occurs in the regime $A \in[0.70,0.80]$ for our parameters. Recalling the fact the discreteness takes an essential effect on the DS motion in this regime, we may denote another parameter,

$$
\kappa=\frac{8 \pi^{3} \sinh ^{2}(\mu)}{\mu^{3} \sinh \left(\pi^{2} / \mu\right) \sqrt{F(\mu)} \sigma},
$$

as a ratio of the amplitude of the discreteness-generated force to the standard deviation of the randomness-generated force (the formula $F(\mu)$ can still be a good approximation near $\mu \sim$ 1 [16]). In Fig. 4(b) the variation of $\kappa$ with $A$ is also displayed, which clearly shows that this parameter surpasses the value $\kappa \sim 0.1$, increasing to $\sim 1$ in the above crossover regime (the discreteness- and randomness-generated forces become comparable), in contrast to the interval for the small DSs where we have $\kappa \ll 1$ (several orders smaller). Such a condition further confirms the effect of discreteness-randomness interplay in shrinking scatters of the time-averaged observables, comparing with the Richardson-type diffusion where the disorder dominates. For much larger DSs (e.g., with $A>1$ ), the soliton is tightly "stuck" in the lattice due to the big PN barrier induced by discreteness, and it would take too long a time for the weak randomness to drive a substantial displacement, which is not involved in the current work.

\section{CONCLUSIONS}

In summary, we have studied the transport of discrete solitons driven by a dynamically disordered potential. Two phases of soliton diffusion, depending on the soliton size, are revealed from both of the ensemble- and time-averaged descriptions. For the ensemble-averaged observables, the small solitons whose motion is dominated by disorder present a Richardsontype diffusion with $\left\langle x^{2}(t)\right\rangle \sim t^{3}$, while as $t<10^{6}$ the larger solitons show a transient anomalous diffusion affected by the discreteness-disorder interaction. For the time-averaged observables, both the small and larger solitons suggest a ballistic scaling $\left\langle\overline{\delta^{2}(\Delta)}\right\rangle \sim \Delta^{2}$ for the lag times far less than our computational limit. However, the distribution of the normalized quantity $\xi=\overline{\delta^{2}(\Delta)} /\left\langle\overline{\delta^{2}(\Delta)}\right\rangle$ becomes narrower and symmetric as the soliton grows larger, and its variance drops down for an order of magnitude after the discreteness progressively dominates. Our discussions consequently indicate that the ergodicity is violated for considerably long-time diffusion of the discrete solitons.

These investigations may pave the way for simulating anomalous diffusion (especially for the less-understood hyperdiffusion) by soliton transport in disordered environments. On the other hand, to some degree we expect our results to guide experimental realizations in the relevant nonlinear optical and atomic lattices, which are ideal platforms to study the phenomena of wave spreading and localization [1,2,4,32]. Besides, we could try to extend our studies to the coupled or high-dimensional noisy lattice systems with various forms of nonlinearities, which might be included in future publications.

\section{ACKNOWLEDGMENTS}

The referees are appreciated for their valuable and stimulating comments. This work is supported by the National Natural Science Foundation of China (Grant No. 11902016), by the Fundamental Research Funds for the Central Universities, and by the Zhuoyue Talent Program of Beihang University.

\section{APPENDIX A: VALIDATION OF THE DS APPROXIMATION}

The DNLS Eq. (1) $\left[\epsilon_{n}(t) \equiv 0\right]$ is a nonintegrable model with numerically iterated DS solutions [2-4]. Its integrable counterpart, the Ablowitz-Ladik (AL) model, permits an exact soliton solution with a similar form of Eq. (3).

Hereby we will show that Eq. (3) is also able to well approximate the DS solutions for the DNLS model when the parameter $\mu<1$. In fact, a relevant comparison of the DNLS DSs with those of the AL model can be found in Ref. [4].

TABLE I. The fitting parameter $\mu$ and fitting error $\theta$ for the DSs with $A=0.40-0.80(v=1)$.

\begin{tabular}{lccccc}
\hline \hline$A$ & 0.40 & 0.50 & 0.60 & 0.70 & 0.80 \\
$\mu$ & 0.28 & 0.35 & 0.41 & 0.48 & 0.54 \\
$\theta$ & $2.9 \times 10^{-4}$ & $7.3 \times 10^{-4}$ & $1.5 \times 10^{-3}$ & $2.8 \times 10^{-3}$ & $4.8 \times 10^{-3}$ \\
\hline \hline
\end{tabular}



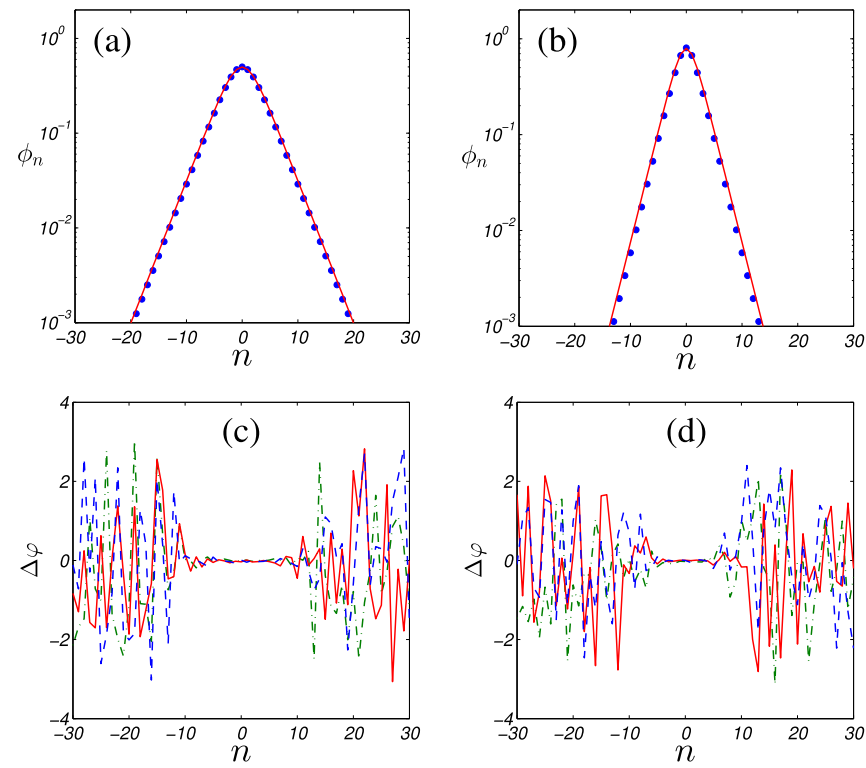

FIG. 5. The DS profiles at $t=0$ for (a) $A=0.50$ and (b) $A=$ $0.80(v=1)$. Blue circles represent the numerically iterated profiles $\phi_{1, n}$ and red solid lines correspond to the fitted profiles $\phi_{2, n}$ with Eq. (3). The phase gradient $\Delta \varphi$ for three realizations of DSs at $t=10^{6}$, with (c) and (d) respectively corresponding to the cases of $A=0.50$ and $A=0.80$ (the coordinate origin is located on the DS center).

Firstly, the shape of the numerically iterated DSs is fitted by using Eq. (3). We denote the relative fitting error as

$$
\theta=\frac{\left\|\phi_{1, n}-\phi_{2, n}\right\|_{2}^{2}}{\left\|\phi_{1, n}\right\|_{2}^{2}}=\frac{1}{P}\left\|\phi_{1, n}-\phi_{2, n}\right\|_{2}^{2},
$$

where $\phi_{1, n}$ is the numerically iterated profile of the DSs, $\phi_{2, n}$ is the fitted profile satisfying $\phi_{2, n}=\sqrt{2 / v} \sinh (\mu) / \cosh [\mu(n-$ $x)$ ], and $\|\cdot\|_{2}$ represents the $L^{2}$ norm of a vector. We look for the fitting parameter $\mu$ that minimizes this error, i.e.,

$$
\mu=\arg \min _{\mu \in \mathbb{R}^{+}} \theta .
$$

The fitting results for typical DSs, including those used in this paper, are listed in Table I. We see that the DS profiles are well fitted by the assumption (3) with very small relative errors. Two DS profiles at $t=0$, comparing with their fitted
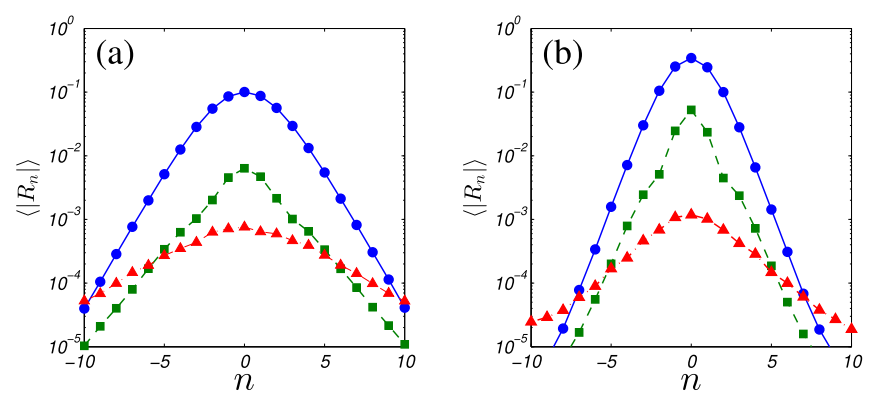

FIG. 6. The ensemble-averaged terms $\left\langle\left|R_{n}\right|\right\rangle$ at $t=10^{6}$ for the cases of (a) $A=0.50$ and (b) $A=0.80$ ( $v=1$ and $\sigma=0.0002$ ). From upper to lower curves, blue circles, green squares, and red triangles respectively correspond to the terms $R_{0, n}, R_{1, n}$, and $R_{2, n}$ (the coordinate origin is at the DS center).
TABLE II. The values of $\rho$ at $t=10^{6}(\nu=1$ and $\sigma=0.0002)$.

\begin{tabular}{ccccc}
\hline \hline$A$ & 0.50 & 0.60 & 0.70 & 0.80 \\
$\rho$ & 0.038 & 0.039 & 0.041 & 0.045 \\
\hline \hline
\end{tabular}

configurations, are also illustrated in Figs. 5(a) and 5(b). Due to very weak radiation, the core of the DSs is nearly unchanged [see Fig. 1(a)], and thus the approximation Eq. (3) of DS profiles can be effective for a considerably long time.

On the other hand, Eq. (3) indicates that the assumed DS has a constant phase difference in consecutive sites, i.e., $\Delta \varphi_{n}=\varphi_{n}-\varphi_{n-1}=$ const, where $\varphi_{n}=\arg \psi_{n}$. We checked the phase gradient up to $t=10^{6}$ for all our computational realizations and found that the core of the DS is characterized by an approximately constant $\Delta \varphi$. Three realizations are respectively plotted in Figs. 5(c) and 5(d) for the cases of $A=0.50$ and $A=0.80$. This shows the fact that Eq. (3) reasonably describes the phase of the DS core as well.

\section{APPENDIX B: COMMENTS ON THE PERTURBATION APPROACH}

An effective way to study the moving DSs for the DNLS model is considering its nonintegrable term as a perturbation to the AL model and applying the soliton perturbation theory [10,60-62]. This method is equivalent to the variational approach for collective coordinates [61,63].

We rewrite Eq. (1) as the following:

$$
\begin{gathered}
i \frac{\partial \psi_{n}}{\partial t}+\left(\psi_{n-1}+\psi_{n+1}\right)+\underbrace{\frac{v}{2}\left|\psi_{n}\right|^{2}\left(\psi_{n-1}+\psi_{n+1}\right)}_{R_{0, n}} \\
=\underbrace{\frac{v}{2}\left|\psi_{n}\right|^{2}\left(\psi_{n-1}+\psi_{n+1}-2 \psi_{n}\right)}_{R_{1, n}}+\underbrace{\epsilon_{n}(t) \psi_{n}}_{R_{2, n}} .
\end{gathered}
$$

For the core of the DSs, the term $\left|R_{2, n}\right|$ is much smaller than $\left|R_{0, n}\right|$ due to the very weak random potential, and meanwhile $\left|R_{1, n}\right|$ for $\mu<1$ is at least an order of magnitude less than $\left|R_{0, n}\right|$ up to $t=10^{6}$ (see two showcases in Fig. 6).

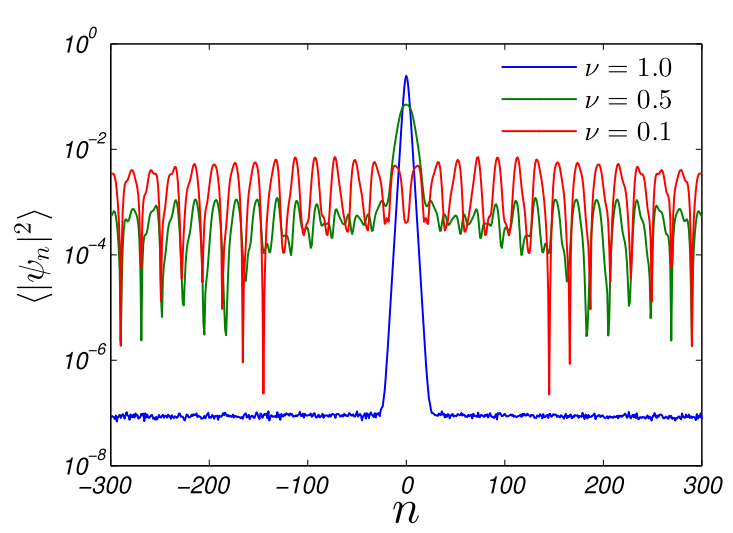

FIG. 7. The ensemble-averaged wave packets $\left\langle\left|\psi_{n}\right|^{2}\right\rangle$ at $t=10^{3}$ for $v=1.0$ (blue line), $v=0.5$ (green line), and $v=0.1$ (red line). The DS with $A=0.50$ was used as the initial condition and the parameter $\sigma=0.0002$ was considered. 
Recombination of the nonlinear terms leads the 1.h.s. of Eq. (B1) to be the form of the AL model and the r.h.s. to be a relatively small perturbation for the sites within the DS core. Therefore it would be valid to employ the perturbation theory for the AL model $[60,61]$ to approximately obtain Eq. (4).

\section{APPENDIX C: RADIATION AND NONLINEARITY}

The total mass of very small linear waves radiated from the core of DSs is calculated by using the numerical data of $\left\langle\left|\psi_{n}\right|^{2}\right\rangle$ up to $t=10^{6}$, which is denoted as $M_{r}$. The ratio of $M_{r}$ to the conserved mass $P$, i.e., $\rho=M_{r} / P$, can describe the radiation level. Table II gives the values of $\rho$ for the cases of
$A=0.50-0.80$, which reveals that the radiation mass is quite small even up to our computational limit. We also estimated the contribution of radiation mass in numerically calculating the center of mass of DS and found that it contributed no more than $6 \%$ of the final result for $A=0.50-0.70$ and less than $7 \%$ for $A=0.80$.

On the other hand, we stress that in this study a relatively strong nonlinearity is considered, which ensures that even the small DSs (e.g., for $A=0.50$ ) keep their solitonic identity no shorter than $t=10^{6}$, in contrast with previous works on the wave packet spreading by weak nonlinearity [13,14]. In Fig. 7 we show that the soliton structure may be broken up at an early time if the strength of nonlinearity is decreased by an order of magnitude, resulting a wide spread of the wave packets that is completely different from the soliton diffusion.
[1] F. Lederer, G. I. Stegeman, D. N. Christodoulides, G. Assanto, M. Segev, and Y. Silberberg, Discrete solitons in optics, Phys. Rep. 463, 1 (2008).

[2] S. Flach and A. V. Gorbach, Discrete breathers-advances in theory and applications, Phys. Rep. 467, 1 (2008).

[3] P. G. Kevrekidis, The Discrete Nonlinear Schrödinger Equation (Springer-Verlag, Berlin, 2009).

[4] R. Franzosi, R. Livi, G. L. Oppo, and A. Politi, Discrete breathers in Bose-Einstein condensates, Nonlinearity 24, R89 (2011).

[5] S. V. Dmitriev, E. A. Korznikova, Yu A. Baimova, and M. G. Velarde, Discrete breathers in crystals, Phys. Usp. 59, 446 (2016).

[6] M. Johansson and P. Jason, Quodons in Mica, Springer Series in Materials Science (Springer, New York, 2015), Vol. 221, pp. 147-178.

[7] L. Hadžievski, A. Maluckov, M. Stepić, and D. Kip, Power Controlled Soliton Stability and Steering in Lattices with Saturable Nonlinearity, Phys. Rev. Lett. 93, 033901 (2004).

[8] U. Naether, R. A. Vicencio, and M. Stepić, Mobility of highpower solitons in saturable nonlinear photonic lattices, Opt. Lett. 36, 1467 (2011).

[9] B. Rumpf, Intermittent movement of localized excitations of a nonlinear lattice, Phys. Rev. E 70, 016609 (2004).

[10] Y. S. Kivshar and D. K. Campbell, Peierls-Nabarro potential barrier for highly localized nonlinear modes, Phys. Rev. E 48, 3077 (1993).

[11] S. Aubry, Discrete breathers: Localization and transfer of energy in discrete Hamiltonian nonlinear systems, Physica D 216, 1 (2006).

[12] F. Abdullaev and J. Garnier, Optical solitons in random media, Prog. Opt. 48, 35 (2005).

[13] A. S. Pikovsky and D. L. Shepelyansky, Destruction of Anderson Localization by Weak Nonlinearity, Phys. Rev. Lett. 100, 094101 (2008).

[14] S. Flach, D. O. Krimer, and C. Skokos, Universal Spreading of Wave Packets in Disordered Nonlinear Systems, Phys. Rev. Lett. 102, 024101 (2009).

[15] I. Vakulchyk, M. V. Fistul, and S. Flach, Wave Packet Spreading with Disordered Nonlinear Discrete-Time Quantum Walks, Phys. Rev. Lett. 122, 040501 (2019).
[16] Z.-Y. Sun, S. Fishman, and A. Soffer, Soliton mobility in disordered lattices, Phys. Rev. E 92, 040903(R) (2015).

[17] Z.-Y. Sun and X. Yu, Transient diffusion and two-regime localization of discrete breatherlike excitations in nonlinear Schrödinger lattice with disorder, Phys. Rev. E 100, 022202 (2019).

[18] L. Levi, Y. Krivolapov, S. Fishman, and M. Segev, Hypertransport of light and stochastic acceleration by evolving disorder, Nat. Phys. 8, 912 (2012).

[19] Y. Krivolapov, L. Levi, S. Fishman, M. Segev, and M. Wilkinson, Super-diffusion in optical realizations of Anderson localization, New J. Phys. 14, 043047 (2012).

[20] A. Amir, Y. Lahini, and H. B. Perets, Classical diffusion of a quantum particle in a noisy environment, Phys. Rev. E 79, 050105(R) (2009).

[21] A. Iomin, Hyperdiffusion of quantum waves in random photonic lattices, Phys. Rev. E 92, 022139 (2015).

[22] A. Radosavljević, G. Gligorić, P. P. Beličev, A. Maluckov, and M. Stepić, Light propagation in binary kagome ribbons with evolving disorder, Phys. Rev. E 96, 012225 (2017).

[23] C. D’Errico, M. Moratti, E. Lucioni, L. Tanzi, B. Deissler, M. Inguscio, G. Modugno, M. B. Plenio, and F. Caruso, Quantum diffusion with disorder, noise and interaction, New J. Phys. 15, 045007 (2013).

[24] Y. V. Kartashov, V. A. Vysloukh, and L. Torner, Brownian soliton motion, Phys. Rev. A 77, 051802(R) (2008).

[25] L. M. Aycock, H. M. Hurst, D. K. Efimkin, D. Genkina, H.-I. Lu, V. M. Galitski, and I. B. Spielman, Brownian motion of solitons in a Bose-Einstein condensate, Proc. Natl. Acad. Sci. USA 114, 2503 (2017).

[26] V. Folli and C. Conti, Frustrated Brownian Motion of Nonlocal Solitary Waves, Phys. Rev. Lett. 104, 193901 (2010).

[27] A. Piccardi, S. Residori, and G. Assanto, Nonlocal soliton scattering in random potentials, J. Opt. 18, 07LT01 (2016).

[28] Z.-Y. Sun and X. Yu, Transport of nonautonomous solitons in two-dimensional disordered media, Ann. Phys. (Berlin) 529, 1600323 (2017).

[29] Y. V. Kartashov and V. A. Vysloukh, Anderson localization of solitons in optical lattices with random frequency modulation, Phys. Rev. E 72, 026606 (2005). 
[30] K. Sacha, C. A. Müller, D. Delande, and J. Zakrzewski, Anderson Localization of Solitons, Phys. Rev. Lett. 103, 210402 (2009).

[31] S. Fishman, Y. Krivolapov, and A. Soffer, The nonlinear Schrödinger equation with a random potential: Results and puzzles, Nonlinearity 25, R53 (2012).

[32] Y. Lahini, A. Avidan, F. Pozzi, M. Sorel, R. Morandotti, D. N. Christodoulides, and Y. Silberberg, Anderson Localization and Nonlinearity in One-Dimensional Disordered Photonic Lattices, Phys. Rev. Lett. 100, 013906 (2008).

[33] A rigorous but more complicated derivation of the scaling $\left\langle x^{2}\right\rangle \sim t^{3}$ can be seen in Refs. [34,35] for the Eq. (7)-type model.

[34] L. Golubović, S. Feng, and F.-A. Zeng, Classical and Quantum Superdiffusion in a Time-Dependent Random Potential, Phys. Rev. Lett. 67, 2115 (1991).

[35] M. N. Rosenbluth, Comment on "Classical and Quantum Superdiffusion in a Time-Dependent Random Potential, Phys. Rev. Lett. 69, 1831 (1992).

[36] L. F. Richardson, Atmospheric diffusion shown on a distanceneighbour graph, Proc. R. Soc. Lond. A 110, 709 (1926).

[37] J. P. Gordon and H. A. Haus, Random walk of coherently amplified solitons in optical fiber transmission, Opt. Lett. 11, 665 (1986).

[38] A. M. Obukhov, Description of turbulence in terms of Lagrangian variables, Adv. Geophys. 6, 113 (1959).

[39] U. Frisch, Turbulence, The Legacy of Kolmogorov (Cambridge University Press, Cambridge, 1995).

[40] A. M. Jayannavar and N. Kumar, Nondiffusive Quantum Transport in a Dynamically Disordered Medium, Phys. Rev. Lett. 48, 553 (1982).

[41] G. Falkovich, K. Gawedzki, and M. Vergassola, Particles and fields in fluid turbulence, Rev. Mod. Phys. 73, 913 (2001).

[42] D. A. Kessler and E. Barkai, Theory of Fractional Lévy Kinetics for Cold Atoms Diffusing in Optical Lattices, Phys. Rev. Lett. 108, 230602 (2012).

[43] A. Iomin, Richardson diffusion in neurons, Phys. Rev. E 100, 010104(R) (2019).

[44] Ch. Skokos, E. Gerlach, J. D. Bodyfelt, G. Papamikos, and S. Eggl, High order three part split symplectic integrators: Efficient techniques for the long time simulation of the disordered discrete nonlinear Schrödinger equation, Phys. Lett. A 378, 1809 (2014).

[45] E. Gerlach, J. Meichsner, and C. Skokos, On the symplectic integration of the discrete nonlinear Schrödinger equation with disorder, Eur. Phys. J. Special Topics 225, 1103 (2016).

[46] C. Danieli, B. M. Manda, T. Mithun, and C. Skokos, Computational efficiency of numerical integration methods for the tangent dynamics of many-body Hamiltonian systems in one and two spatial dimensions, Math. Eng. 1, 447 (2019).
[47] R. Metzler, J.-H. Jeon, A. G. Cherstvy, and E. Barkai, Anomalous diffusion models and their properties: Nonstationarity, non-ergodicity, and ageing at the centenary of single particle tracking, Phys. Chem. Chem. Phys. 16, 24128 (2014).

[48] E. Barkai, Y. Garini, and R. Metzler, Strange kinetics of single molecules in living cells, Phys. Today 65, 29 (2012).

[49] I. M. Sokolov, Models of anomalous diffusion in crowded environments, Soft Matter 8, 9043 (2012).

[50] Y. He, S. Burov, R. Metzler, and E. Barkai, Random Time-Scale Invariant Diffusion and Transport Coefficients, Phys. Rev. Lett. 101, 058101 (2008).

[51] Y. Meroz, I. M. Sokolov, and J. Klafter, Subdiffusion of mixed origins: When ergodicity and nonergodicity coexist, Phys. Rev. E 81, 010101 (2010).

[52] A. Godec and R. Metzler, Finite-Time Effects and Ultraweak Ergodicity Breaking in Superdiffusive Dynamics, Phys. Rev. Lett. 110, 020603 (2013).

[53] Y. Meroz and I. M. Sokolov, A toolbox for determining subdiffusive mechanisms, Phys. Rep. 573, 1 (2015).

[54] A. Lubelski, I. M. Sokolov, and J. Klafter, Nonergodicity Mimics Inhomogeneity in Single Particle Tracking, Phys. Rev. Lett. 100, 250602 (2008).

[55] A. G. Cherstvy and R. Metzler, Ergodicity breaking and particle spreading in noisy heterogeneous diffusion processes, J. Chem. Phys. 142, 144105 (2015).

[56] M. Schwarzl, A. Godec, and R. Metzler, Quantifying nonergodicity of anomalous diffusion with higher order moments, Sci. Rep. 7, 3878 (2017)

[57] I. Bronstein, Y. Israel, E. Kepten, S. Mai, Y. Shav-Tal, E. Barkai, and Y. Garini, Transient Anomalous Diffusion of Telomeres in the Nucleus of Mammalian Cells, Phys. Rev. Lett. 103, 018102 (2009).

[58] J. Spiechowicz and J. Łuczka, Diffusion anomalies in ac-driven Brownian ratchets, Phys. Rev. E 91, 062104 (2015).

[59] J. Spiechowicz, J. Łuczka, and P. Hänggi, Transient anomalous diffusion in periodic systems: Ergodicity, symmetry breaking and velocity relaxation, Sci. Rep. 6, 30948 (2016).

[60] Ch. Claude, Yu. S. Kivshar, O. Kluth, and K. H. Spatschek, Moving localized modes in nonlinear lattices, Phys. Rev. B 47, 14228 (1993).

[61] D. Cai, A. R. Bishop, and N. Grønbech-Jensen, Perturbation theories of a discrete, integrable nonlinear Schrödinger equation, Phys. Rev. E 53, 4131 (1996).

[62] K. Kundu, Perturbative study of classical Ablowitz-Ladik type soliton dynamics in relation to energy transport in $\alpha$-helical proteins, Phys. Rev. E 61, 5839 (2000).

[63] N. R. Quintero, F. G. Mertens, and A. R. Bishop, Generalized traveling-wave method, variational approach, and modified conserved quantities for the perturbed nonlinear Schrödinger equation, Phys. Rev. E 82, 016606 (2010). 